Archives

22 | 1999

Réflexions historiographiques

\title{
La société comme un tout : sur trois formes d'analyse de la totalité sociale
}

\section{Bernard Lepetit}

\section{OpenEdition}

Journals

Édition électronique

URL : http://journals.openedition.org/ccrh/2342

DOI : $10.4000 /$ ccrh.2342

ISSN : $1760-7906$

Éditeur

Centre de recherches historiques - EHESS

Édition imprimée

Date de publication : 20 avril 1999

ISSN : 0990-9141

Référence électronique

Bernard Lepetit, «La société comme un tout: sur trois formes d'analyse de la totalité sociale », Les Cahiers du Centre de Recherches Historiques [En ligne], 22 I 1999, mis en ligne le 17 janvier 2009, consulté le 19 avril 2019. URL : http://journals.openedition.org/ccrh/2342 ; DOI : 10.4000/ccrh.2342

Ce document a été généré automatiquement le 19 avril 2019

Article L.111-1 du Code de la propriété intellectuelle. 


\title{
La société comme un tout : sur trois formes d'analyse de la totalité sociale
}

\author{
Bernard Lepetit
}

\section{NOTE DE L'ÉDITEUR}

Bernard Lepetit est décédé accidentellement en 1996 : republier ses écrits est une façon de tenter de « franchir cet absurde silence ».

1 Comment faire une histoire totale ? Autrement dit, comment rendre compte, à partir de la conduite individuelle des acteurs (du côté de l'objet) et d'enquêtes empiriques nécessairement limitées (du côté des méthodes) de la totalité sociale et des processus macro-historiques? Au moins dans l'historiographie française, la réponse ne va plus de soi. D'une part, les historiens qui se réclament des Annales sont fréquemment accusés, depuis plusieurs années, d'avoir oublié le projet d'histoire totale qui était celui des fondateurs de la revue au profit d'un émiettement des méthodes et des pôles d'intérêt de la discipline. D'autre part, l'histoire sérielle fondée sur l'analyse statistique de données nombreuses et agrégées semble entrée dans une phase de rendements décroissants, et une micro-histoire développant une exploitation intensive d'objets très étroitement circonscrits vient offrir un modèle historiographique alternatif dont il paraît facile de se réclamer ${ }^{1}$.

2 Pourtant, ni le réarmement des anciennes manières de faire, ni le basculement vers le nouveau paradigme ne me paraissent des réponses adéquates aux incertitudes du moment. Les deux approches rencontrent, chacune pour leur part, des difficultés difficiles à surmonter qui encouragent à prêter attention aux solutions plus inventives élaborées dans d'autres disciplines. La présente contribution, conçue comme une sorte de compte-rendu de lecture, n'a pas d'autre but que de souligner les difficultés des méthodes 
historiques concurrentes et de suggérer d'autres voies, qui vaudraient d'être explorées par les historiens.

\section{L'échec d'une totalisation cartésienne}

3 En 1941, dans une conférence donnée devant les élèves de l'École normale supérieure, Lucien Febvre a explicité les raisons de l'emploi, par Marc Bloch et lui-même, de l'adjectif « social » dans le titre de la revue qu'ils avaient fondée douze ans plus tôt :

Nous étions d'accord pour penser que, précisément, un mot aussi vague que «social » semblait avoir été créé et mis au monde par un décret nominatif de la providence historique, pour servir d'enseigne à une revue qui prétendait ne pas s'entourer de murailles... Il n'y a pas d'histoire économique et sociale, il y a l'histoire tout court, dans son unité. L'histoire qui est sociale tout entière, par définition ${ }^{2}$.

4 Ceci étant posé, l'analyse de la totalité sociale, de par sa globalité même, est une opération intellectuelle difficile: tout se joue dans les modalités de sa mise en œuvre. Celles qui ont été communément pratiquées par l'historiographie française reposent sur la décomposition préalable de l'espace, du temps ou des domaines de la réalité humaine : la connaissance du tout était censée naître de celle, plus accessible, de ses parties.

Des motifs d'opportunité expliquent en partie que la monographie locale ait constitué pendant plus de vingt ans le genre dominant de la recherche historique française : une ville, un département, une province fournissaient un sujet aux dimensions d'un fonds d'archives localisé, et il apparaissait plus facile de convertir une compétence scientifique régionale en autorité universitaire de même échelle. Mais la monographie définie sur des bases géographiques trouvait sa justification fondamentale dans une croyance épistémologique commune : le savoir global progresse par accumulation de connaissances locales. Rassembler encore quelques bonnes monographies régionales et regrouper leurs données pour résoudre la question d'ensemble, telle est la démarche préconisée aussi bien par Lucien Febvre en 1922 que par Ernest Labrousse après la Seconde Guerre mondiale ${ }^{3}$. Le projet, pourtant, n'aboutit pas. L'étude des processus généraux - l'anoblissement dans la société française d'Ancien Régime, ou la révolution industrielle en Europe, par exemple - ne résulte pas de la combinaison des analyses précédentes; elle se développe, dans un autre cadre, à une autre échelle, avec d'autres méthodes et d'autres indicateurs. Quant aux manuels d'histoire générale, s'ils reprennent fréquemment des éléments du savoir positif monographique, c'est pour leur donner surtout un statut illustratif.

6 Tout empêche que ce projet cumulatif puisse aboutir : l'isolement des chercheurs qui mènent individuellement leurs travaux; l'évolution des problématiques au fur et à mesure que les recherches s'élaborent; l'absence de réflexion sur la signification (variable d'une monographie à l'autre) des limites adoptées pour l'objet et donc sur la manière dont ils s'articulent à d'autres objets d'échelle différente. Espérer accéder à des considérations générales de cette manière, en effectuant la somme des constats particuliers, c'est confondre la découpe des pièces du puzzle avec les contours du dessin que celui-ci représente et que, précisément, elles ont pour effet de masquer. Ainsi, dans cette procédure de recherche, le local et le global ne communiquent pas, ou mal. L'accès à l'histoire totale par le truchement d'une décomposition géographique de l'univers historique se heurte à des difficultés méthodologiques qui conduisent à réitérer des 
descriptions monographiques qui trouvent en elles-mêmes leur propre finalité, et tendent à réifier leur objet.

7 Deux objections viennent pourtant à l'esprit qui réduiraient à peu de choses cette difficulté. La première soulignerait que la ville ou la région ne sont pas seulement des catégories spatiales de l'analyse. Elles sont également des êtres géographiques que des différences dans le paysage naturel ou culturel, que des flux de relations économiques ou sociales délimitent, structurent et matérialisent. Partant, c'est à juste titre qu'on peut les étudier pour elles-mêmes : leur réification est licite puisque chacune des catégories de l'analyse trouve son exact correspondant dans le réel. La seconde objection est de nature différente: le changement d'échelle auquel correspond la monographie locale tire sa légitimité de l'uniformité des situations. Elle a un statut de sondage. À son échelle, le chercheur y développe une histoire totale qui vaut pour un exemple plus vaste: ce que l'analyse de Beauvais, de Lyon ou du Languedoc donne à voir, c'est le système socioéconomique d'Ancien Régime tout entier. La totalisation ne s'opère plus par addition mais par homologie. Il faut examiner successivement ces deux objections.

J'analyserai, pour faire face à la première, les modalités de mise en œuvre des catégories temporelles par la recherche historique du second après-guerre ${ }^{4}$. Comme l'échelle spatiale, l'échelle chronologique est un élément déterminant pour la lecture d'un phénomène. Mais les figures du temps et de l'espace n'offrent pas les mêmes formes de structuration de l'expérience : au temps uniforme du calendrier, décomposable en unités de durée variable mais homogène et répétitif, s'oppose l'espace hétérogène et particularisé de la carte. En apparence, la matérialité des lieux offre aux opérations de découpage de l'espace des points d'appui et des lignes de différenciation plus solides que le déroulement linéaire du temps n'en offre aux découpages chronologiques : quels seront les équivalents temporels de la ville, de la région ou de l'espace national ? Le réalisme possible des catégories spatiales d'analyse ne trouve pas sa contrepartie dans l'ordre temporel. L'indicateur est donc pertinent pour l'usage que je souhaite en faire : montrer que l'usage des catégories analytiques est fondé sur une attitude épistémologique semblable et qu'elle débouche sur des difficultés du même ordre.

9 Pour «l'histoire historisante » que dénonçaient les fondateurs de Annales, l'événement constituait l'unité temporelle que l'exploration des archives permettait de restituer ; puis, la chronique formait la totalité dont la construction, par concaténation des faits tenus pour vrais, épuisait la description historique. L'explication progressait par accumulation d'événements comme de détails nouveaux. L'historien convié par « l'école des Annales » à un travail de compréhension adopte une démarche opposée. Chaque moment, quelle que soit sa durée, combine une pluralité de temps sociaux dont chacun se déroule selon des rythmes et à une échelle qui lui est propre. L'explication résulte d'un processus d'identification et de désemboîtement les unes des autres de ces temporalités multiples. Le procédé ne postule rien quant à la durée de la séquence chronologique à expliquer: l'époque de Philippe II et la crise révolutionnaire du printemps 1789 relèvent du même type d'analyse qui procède par feuilletage et non par agglomération ${ }^{5}$. Mais on voit bien que le renversement ne touche pas seulement la démarche. Elle affecte aussi le statut des objets temporels concernés : l'événement (au sens historique, sans rien postuler sur sa durée) constitue maintenant la totalité, et les multiples chroniques au sein desquelles il s'inscrit forment les parties dont les modalités de la combinaison font explication. Voyons ce qui joue dans ce processus explicatif où décomposition et corrélation sont les maîtres-mots. 
10 Dans le désordre apparent du particulier, un ordre se dégage, qui est celui du rapprochement des séries chronologiques d'abord individualisées. Le prix du blé des principales villes du Bassin Parisien qui évoluent dans le même sens et en même temps signalent l'existence autour de la capitale d'une région économique unifiée, de la même manière que les nombres rapprochés de mouvements de navires dans les grands ports méditerranéens puis atlantiques signalent le fonctionnement des économies-monde. L'évolution conjointe des courbes démographiques et des prix des aliments de base donne à lire les modalités du système d'équilibre des populations et des ressources. Les mouvements opposés des salaires, des rentes et des profits marquent le fonctionnement d'une formation socio-économique et détermine ses soubresauts politiques. Pour qui sait les lire, les courbes qui rendent compte des fluctuations en plusieurs lieux de grandeurs économiques, sociales, culturelles, politiques, sont un moyen d'accès à la globalité. Leurs corrélations mêmes sont le signe et la garantie que la réalité dont elles sont la mesure forme système. Elles s'inscrivent ainsi dans un projet d'histoire totale. Elles n'en permettent guère pour autant l'accomplissent, comme je voudrais le montrer maintenant.

11 Parmi la pluralité des temps, deux dimensions on le sait ont été généralement privilégiées: celle des tendances séculaires de la longue durée et celle des diverses oscillations cycliques qui couvrent des périodes qui s'étagent de quelques années (Kitchin) à un demi-siècle environ (Kondratiev). D'un côté la structure, « réalité que le temps use mal et véhicule très longuement », de l'autre le "récitatif » de la conjoncture 6 . Le couplage de ces deux catégories temporelles a longtemps fourni un brevet de scientificité et fondé l'ordre d'exposition des résultats de la recherche ${ }^{7}$. Dans cette approche, c'est à la technique statistique que revient la charge de réduire la complexité de la totalité : la décomposition des séries chronologiques appartient au bagage de tout historien ${ }^{8}$. On connaît les étapes traditionnelles les plus usuelles. Elles enchaînent la mise en évidence du mouvement le plus long, son élimination, la mise en évidence du mouvement d'une durée immédiatement inférieure au précédent, et ainsi de suite. Une représentation graphique vient généralement illustrer le discours : chaque mouvement s'y enroule sur l'axe formé par le mouvement de durée immédiatement supérieure ${ }^{9}$. Cette procédure appelle deux remarques.

12 D'une part, elle établit de fait une hiérarchie entre les mouvements de durées différentes. Chacun d'eux a, par rapport au mouvement de longueur immédiatement supérieure le caractère d'un reste: le cycle Kondratiev par exemple, c'est ce qui reste lorsque la tendance séculaire a été éliminée. Le statut de l'événement (au sens traditionnel cette fois), simple agitation de surface, révélateur de structures ou de conjonctures dont il n'est que la manifestation visible des effets, en témoigne : le plus fondamental est du côté du temps long. Mais rien d'autre que la technique statistique et l'ordre dans lequel elle isole les mouvements ne vient justifier une hiérarchie. Celle-ci ne trouve sa source ni dans une description phénoménologique (celle des échelles de la conscience temporelle des acteurs par exemple), ni dans une analyse théorique des processus : sa logique est entièrement extérieure au système dont elle assure fournir, par son mouvement de décomposition/ recomposition, les clés. D'autre part, les formes de l'articulation des temporalités de durées différentes n'est pas non plus pensée. « Cycles de Kitchin, de Juglar, Kondratiev et phases se superposent sans contestation possible » : à vrai dire, ils ne se superposent que sur les graphiques que Pierre Chaunu par exemple a manifestement en tête lorsqu'il écrit 10. Car pour le reste, ils ne communiquent pas. Si, comme l'a suggéré Ernest Labrousse, 
une formation sociale possède la conjoncture de ses structures, le renouvellement des caractères structuraux doit trouver son origine dans d'autres sources que dans le mouvement conjoncturel; mais de telles sources n'existent pas. Deux conséquences en résultent sur le plan historiographique. La première est un repli alternatif soit vers une histoire conjoncturelle à la manière de Labrousse, soit vers la plus longue durée de «l'histoire immobile » naguère prônée par E. Le Roy Ladurie ${ }^{11}$. La seconde est la fortune thématique de la révolution: l'historiographie du second après-guerre en vit de toutes natures, démographique, agricole, industrielle, intellectuelle, politique même. Alors, tout est mutation brusque dans une explication qui ne peut penser le changement que comme une discontinuité radicale entre une structure et la suivante. On verra dans ces deux conduites méthodologiques de fuite un même symptôme: celui d'une incapacité à recomposer la totalité historique, changeante par nature, au terme du processus de décomposition analytique qui était censé la donner à voir. La difficulté est de même type dans l'ordre temporel que dans l'ordre spatial.

13 Reste la seconde objection: la monographie est le microcosme à l'échelle duquel est développée l'histoire totale qui prend en compte à la fois les dimensions économiques, sociales et culturelles de l'expérience humaine. Je partirai, pour l'examiner, de l'histoire sociale. Celle-ci a d'abord été, en France, une étude des structures : il s'agissait de définir, de délimiter et de dénombrer des groupes, d'examiner les liens de domination et de dépendance qui les mettent en rapport, les formes de stratification sociale qui en résultent. La querelle n'est plus guère le moyen par lequel le savoir historique progresse ; il n'en est donc que plus remarquable que la nature des structures sociales d'Ancien Régime a donné lieu, dans les années soixante, à l'une des dernières d'entre elles. Aux tenants de la nature de classe (définie en termes de statut socio-professionnel et de niveaux de fortune) des sociétés d'Ancien Régime regroupés autour d'Ernest Labrousse, s'opposaient les partisans d'une société d'ordre, fondée sur l'estime sociale collective attachée à chaque condition, menés par Roland Mousnier ${ }^{12}$. Ce débat aujourd'hui reste intéressant par les blocages qu'il révèle : je ne reprendrai ici que ceux qui relèvent de l'usage des catégories.

Développée dans les termes précédents, l'analyse des structures est nécessairement tautologique. Qu'on privilégie les hiérarchies de fortunes ou les formes les plus fréquentes de l'inter-mariage, le produit des dépouillements des registres fiscaux ou des archives notariales vient seulement nourrir de données empiriques les catégories prédéterminées. Malheureusement, les classements sont multiples; ils sont à la fois partiellement (ou totalement) inconciliables et tous corroborés par l'observation empirique puisqu'ils l'organisent. Le prix à payer pour sauver l'un ou l'autre de ces classements est particulièrement élevé, même eu égard aux exigences assez limitées de la discipline. L'historien doit dans un même mouvement: invoquer contre les interprétations historiennes concurrentes un simple argument d'autorité (recours à Marx comme théoricien des classes ou au contraire à tel théoricien ancien des ordres); renvoyer au rang des idéologies les classements à travers lesquels les sociétés d'autrefois se pensaient, et soutenir que les visions traditionnelles ne faisaient que masquer «les réalités profondes » du passé ; postuler la simplicité fondamentale du réel, dont la connaissance pourrait progresser par réduction à un principe unique, réifier les catégories analytiques afin de donner la force de l'évidence à la description chiffrée à laquelle se résumait l'analyse sociale ; dénier enfin aux acteurs une capacité créatrice. 
L'une des premières critiques de la démarche sociographique vint de Jean-Claude Perrot. Dans un article publié en 1968, considérant que

[...] les sociétés sont à la fois ce qu'elles pensent être et ce qu'elles ignorent qu'elles sont,

il préconisait d'étudier, plutôt que les structures, les relations sociales. Les cérémonies publiques, les formes associatives, les lieux de rencontre, les manifestations de la violence constituent autant de dimensions de la société citadine dont la description permet d'accéder à la connaissance des sociétés du passé13. Publié en 1975, à la différence de toutes les monographies d'histoire urbaine du moment, son livre sur Caen ne comporte aucune étude particulière des "structures sociales ». Mais l'alternative qu'il propose est différente de celle qu'il suggérait quelques années plus tôt: pas plus que l'analyse des stratifications, la seule analyse des relations sociales n'est susceptible d'épuiser la compréhension des sociétés.

Une lecture perspicace doit sentir que les comportements de la population, la pratique médicale, les processus qui règlent la production, les échanges, l'aménagement des quartiers, décrivent efficacement les fondements de l'histoire sociale ${ }^{14}$.

C'est une manière de retrouver la définition braudélienne de la société comme « ensemble des ensembles » et souligner, par différence, l'écart par rapport à la démarche habituelle. Soit directement, soit en inversant les termes, le débat précédent s'inscrivait en effet dans un modèle marxisant simplifié des catégories. De l'économique au social et du social au culturel, quel que soit l'ordre des déterminations (l'économie en tête pour Labrousse, la société pour Mousnier, la culture pour le dernier Chaunu), une adéquation généralisée est supposée. En conformant par exemple les catégories sociales aux catégories proposées par l'histoire économique, et en inscrivant ensuite les faits politiques ou culturels dans les cases du tableau socio-économique ainsi constitué, on faisait résulter l'histoire globale d'une sorte de totalisation en ligne rendue possible par le fait que les différents éléments qui en ressortissent peuvent être rangés de manière identique. Mais la signification d'une telle totalisation n'est jamais mise à l'épreuve, puisqu'elle se trouve entièrement contenue dans la partition et la hiérarchisation initiales. Simple tautologie, donc: la juxtaposition de plusieurs études partielles (démographie, économie, société, politique, culture) n'aboutit qu'à faire tomber le sujet en ruine.

Déserter le terrain d'une histoire totale dont on apercevait l'impossibilité pouvait devenir plus qu'une tentation. La fragmentation de la discipline en sous-champs autonomes (de la démographie historique à l'histoire des techniques, de l'histoire économique à celle des mentalités), qui furent tenus chacun pour un temps comme un domaine pionnier, vint en témoigner. Comme en témoigne la faveur du refuge offert par une anthropologie culturelle dans laquelle l'analyse des représentations tend à se refermer sur elle-même, et où les discours passés se trouvent à leur tour réifiés. La recomposition de la globalité, à nouveau, s'achève méthodologiquement dans une impasse. Chosifiées, des catégories analytiques simplificatrices ossifient les processus historiques et les démarches intellectuelles qui les donnent à voir. 


\section{Variation d'échelle et expérience des acteurs}

18 En pratiquant l'étude intensive d'objets très limités (un fait divers, un procès, un rituel, un individu presque ordinaire), la microstoria avance depuis plusieurs années des propositions alternatives. Les premières justifications épistémologiques de l'entreprise témoignent pourtant de la prégnance du modèle macro-analytique. D'une part, les microhistoriens prétendent se glisser dans les interstices de l'analyse sérielle, en accédant au vécu et à l'expérience individuelle inaccessible aux études agrégées. D'autre part, ils entendent apporter aux problèmes de la validation de l'analyse des réponses de même nature que celles que l'histoire quantitative était censée trouver dans le maniement des nombres. Les définitions variables données à la notion « d'exceptionnel normal » forgée pour faire face à la question de la représentativité du cas en portent la marque, qu'il s'agisse de plaider la capacité révélatrice ou bien la normalité de l'exception dans les sociétés anciennes ${ }^{15}$. L'accès à la totalité sociale semblait pouvoir s'opérer à ce prix. Mais posé ainsi, le problème ne comportait pas de solution.

Un retour à des pratiques plus anciennes permettra de le comprendre. Au milieu du XIX siècle, en alternative à la statistique sociale qui se développait alors, Frédéric Le Play envisageait, pour l'étude des familles ouvrières, une méthode en trois étapes, qu'il faut rappeler ${ }^{16}$. D'abord, au cours du travail de terrain, il proposait d'observer des faits particuliers concernant une seule famille (ou un très petit nombre d'entre elles). Une fois achevée cette micro-étude, on s'efforçait d'en tirer, par induction, des propositions générales. Enfin, on soumettait ces conclusions au jugement d'experts, le plus souvent des notables locaux : maires, notaires, médecins... Leur particularité était d'appartenir aussi bien à l'univers observé (ils vivaient dans la même communauté humaine que les familles qui faisaient l'objet d'enquêtes) qu'à celui de l'observateur savant (ils entretenaient comme lui, bien que seulement pour des raisons sociales, une distance critique vis à vis des manières d'être des familles ouvrières). La place de ces experts dans le dispositif d'enquête est importante puisqu'ils forment l'instance de validation qui permet de rompre la circularité d'une analyse qui induit d'observations particulières des conclusions générales, sans pouvoir mettre celles-ci à l'épreuve d'autres données que celles qui ont permis de les forger. Mais qui jouera entre le meunier hérétique du $\mathrm{XVI}^{\mathrm{e}}$ siècle et l'historien d'aujourd'hui le rôle de l'expert? La méthode de Le Play est intéressante ici comme indice. La réponse qu'elle apporte à la question de la validation signale a contrario que le problème de la représentativité, préliminaire à toute forme de généralisation dans ce cadre analytique, ne trouve pas de solution en dehors d'un raisonnement probabiliste et de méthodes d'échantillonnage.

C'est du côté de l'anthropologie anglo-saxonne que la microstoria allait trouver les procédures analytiques différentes qui lui permettaient d'échapper à la fascination du paradigme quantitatif. Contre un premier modèle, inspiré des propositions de Clifford Geertz et qui offrait les ressources d'une science interprétative, les historiens italiens élevèrent rapidement un rempart de critiques $^{17}$. L'anthropologie culturelle, on le sait, entend considérer comme un texte signifiant l'ensemble des actions, des comportements, des rites et des croyances qui forment le tissu social, et donne comme tâche aux sciences humaines de déchiffrer le sens du texte. Elle définit la culture comme un monde de symboles partagés, comme les mots et les structures d'une langue qui sont l'horizon de possibilité de toute prise de parole. Accéder à une connaissance générale, dans ce cas, 
consiste à restituer le langage qui est à la disposition des acteurs qui se bornent, dans les situations particulières où ils sont engagés, à l'articuler.

21 Un postulat implicite est fondateur de ce projet anthropologique: la stabilité de la relation qui associe le «texte » de l'action sociale localisée et la «langue» de la culture dont elle est l'expression. "Les systèmes de signes sont partagés comme l'air que nous respirons ", écrit Robert Darnton à la suite de Clifford Geertz; ou bien encore "des grammaires culturelles ont réellement existé ». Bien entendu, chaque pratique sociale et chaque prise de parole sont susceptibles de modifier la composition de l'enveloppe atmosphérique ou les structures grammaticales, mais à l'échelle de l'action humaine, de telles altérations sont négligeables. Dans l'univers des textes, aux yeux de Darnton en particulier, l'égalisation des caractéristiques contextuelles du moment (les manières françaises de penser le monde au $\mathrm{XVIII}^{\mathrm{e}}$ siècle par exemple) est une garantie contre l'interprétation libre et la condition de la généralisation. L'absence d'autonomie des acteurs sociaux et la saturation interprétative des schémas analytiques sont les deux caractères qui résultent de ce postulat et qui justifient le rejet du modèle par la microstoria. Parce que le contexte qui confère du sens au "texte " est, à l'échelle de l'observation, un invariant, l'analyse porte davantage attention au sens fixé par le « texte » qu'aux processus sociaux, et particulièrement aux conflits d'interprétation, qui aboutissent à sa fixation. Parce que le texte donne à voir le contexte et que le contexte donne du sens au texte, l'analyse interprétative s'achève dans la circularité :

[...] e insomma un processo circolare in cui i criteri di verita e di rilevanza, tutti chiusi nell' attivita ermeneutica costitutiva appaiono... troppo arbitrari.

Le renversement analytique impliqué par ces objections est double. Il aboutit à nier la permanence au profit du changement; il amène sur le devant de la scène, auparavant tout occupée par l'activité interprétative du chercheur, les capacités et les efforts de déchiffrement du monde des acteurs du passé.

« Histoire d'un exorciste ", « itinéraires ouvriers », « naissance d'un langage corporatif »: les sous-titres donnés à des livres qui appartiennent au programme de la micro-histoire dessinent une même structure analytique. Changement du monde paysan et des rapports de pouvoir au XVII ${ }^{\mathrm{e}}$ siècle, modification des aspects et des cadres de la solidarité collective dans une capitale d'Ancien Régime, dynamiques familiales et individuelles de l'intégration ouvrière en ville : c'est un tableau en mouvement qui est restitué à chaque fois ${ }^{18}$. Aucun de ces travaux ne juxtapose des coupes temporelles régulièrement espacées pour faire l'inventaire de leurs ressemblances et de leurs différences afin d'en déduire le processus à l'œuvre. Mais aucun n'est construit non plus comme une chronique: ni l'exhaustivité du rendu, ni la linéarité de la narration n'appartiennent à leurs ambitions. Ce n'est pas l'enchaînement des épisodes mais celui des points de vue analytiques et des modalités successives de l'observation qui commandent leur développement. Explicitement organisés selon des protocoles raisonnés d'étude, ils répondent à la définition de ce qui pourrait être une histoire expérimentale. L'analyse du changement n'y est pas visée parce que le temps constituerait la préoccupation particulière de l'histoire au sein des sciences de l'homme, mais parce que la société est dynamique par nature, et parce que la capacité à rendre compte de l'évolution est un instrument de validation des modèles.

$23 \mathrm{Si}$, dans le cadre d'une histoire expérimentale (ou d'une histoire problème si l'on veut), l'objet historique est construit et non pas donné par avance, c'est la démarche de la recherche qui le porte au jour et l'explicite ${ }^{19}$. Mais en même temps, les deux processus, 
celui de l'évolution du fonctionnement social et de son élucidation, ne sont pas séparables. Le modèle historique se trouve soumis à deux niveaux de validation. Chacun de ses chaînons explicatifs est exposé localement à l'épreuve des observations empiriques correspondantes. Il est ensuite, dans son ensemble, confronté au démenti éventuel de la dynamique sociale: les processus qu'il explicite tirent leur validité de leur non contradiction avec le changement social observé. Processus et expérience : d'une certaine manière, la généralisation s'opère par analogie. La correspondance entre les évolutions prévues par le modèle et les processus observés permet d'appliquer au fonctionnement social passé les principes explicatifs (localement éprouvés empiriquement) dont l'assemblage forme le modèle.

La micro-histoire s'oppose au geertzisme et à ses avatars historiographiques sur un second point, comme on l'a dit: l'attention portée aux capacités interprétatives des acteurs. Les modèles alternatifs lui sont alors fournis par une anthropologie sociale moins attentive aux découpes structurelles de la société qu'aux représentations et aux rôles sociaux, et aux processus de structuration de la société qu'ils induisent par leur interaction. Mais la microstoria s'installe avec ces modèles sur des positions peu conformes à celles que certaines lectures de son programme lui ont attribuées ${ }^{20}$. Un outil d'analyse et une grille théorique fournissent à la microstoria les moyens de la valorisation des acteurs. Les méthodes de la network analysis permettent de reconstruire les réseaux de relations des individus et des familles. Ces réseaux résultent de l'espace d'expérience sociale de chacun et en dessinent l'horizon. Leur identification permet de restituer les formes du regroupement social à partir de la multiplicité des pratiques individuelles. Les éléments théoriques les plus importants sont trouvés chez l'anthropologue norvégien Frederik Barth. La micro-histoire lui emprunte le modèle d'un individu actif et rationnel, opérant pour sa part des choix dans un univers caractérisé par des incertitudes et des contraintes qui dépendent en particulier de la distribution inégale des capacités individuelles d'accès à l'information. De l'ensemble des choix individuels résultent des processus macroscopiques, comme par exemple la pénétration de l'idéologie fasciste dans les milieux ouvriers turinois au $\mathrm{xx}^{\mathrm{e}}$ siècle, ou bien la consolidation variable des corporations de métiers et la formation de l'État moderne trois siècles plus tôt.

25 L'importance différente des ressources dont disposent les acteurs et la diversité de l'étendue des champs dans lesquels ils sont susceptibles d'agir sont parmi les traits essentiels du panorama social, et forment les sources principales de sa modification. La variation d'échelle n'est pas l'apanage du chercheur ni principalement le produit du processus de construction de la recherche: il est d'abord le lot des acteurs. La manipulation délibérée du jeu des échelles a pour fonction d'identifier les systèmes de contextes dans lesquels s'inscrivent les jeux sociaux. L'ambition de cette cartographie dynamique est de repérer et de dessiner, dans leur variété, un ensemble de cartes qui correspondent à autant de territoires sociaux. Mais quant au principe du fonctionnement social, il est pour sa part unique et ne privilégie qu'une seule échelle, celle du microscopique à laquelle opèrent les processus causaux dont dépendent tous les autres. Ainsi s'organise dans les travaux de micro-histoire sinon une contradiction du moins une tension entre une démarche très attentive aux procédures de recherche qui font apparaître des objets historiques inédits en faisant varier l'échelle de l'observation, et le rôle de sanction finale qu'ils accordent à l'expérience individuelle des acteurs du passé. l'accommodement de l'optique possède un double statut : il résulte de la combinaison de 
milliers de situations particulières et en même temps il leur donne sens à toutes. Par exemple, l'évolution de l'État moderne au XvII ${ }^{\mathrm{e}}$ siècle s'est jouée dans des milliers de villages comme celui de Santena, en Piémont, mais en même temps le modèle qui est donné de cette évolution assure qu'il ne sera pas nécessaire de reproduire des milliers de fois l'expérience de Santena pour s'assurer de la valeur générale du cas. L'ensemble des contextes construit au cours de l'expérimentation historiographique est à la fois cadre le plus englobant et niveau de généralisation. Mais la question de savoir si celui qui a été restitué est complet, ou même s'il est le seul envisageable, est sans solution. Invoquer l'expérience des acteurs semble un moyen de rompre une telle incertitude. Un perspectivisme méthodologique vient s'achever dans une forme de réalisme épistémologique.

Tout ce qui est important est macro-économique, tout ce qui est fondamental est micro-économique,

peut-être la microstoria pourrait-elle adopter la formule chère à l'économiste SergeChristophe Kolm. Les micro-historiens contribueraient alors à faire émerger la figure inédite en histoire de l'opposition entre deux modèles conceptuels du social alternatifs, aux objectifs et aux schémas interprétatifs divergents, l'un micro-et l'autre macro-analytique. Certains blocages dénoncés en économie et en sociologie encouragent à explorer d'autres voies. Une sociologie de l'action ou l'économie des conventions proposent aujourd'hui des modèles explicatifs qui refusent ces oppositions simplificatrices. L'exposé d'une démarche plus que de ses résultats, que je poursuis ici, m'autorise à continuer à ne proposer que des fiches de lecture, rédigées sous l'éclairage de la question de l'accès à la totalité sociale.

\section{Conventions générales et accords locaux}

Luc Boltanski et Laurent Thévenot proposent, dans une série d'articles et d'ouvrages, de considérer les actions humaines comme une suite de situations où les acteurs, engagés dans l'échange interpersonnel, mobilisent leurs compétences pour justifier leurs positions ${ }^{21}$. Refusant de considérer aussi bien l'individu abstrait mis en scène par l'économie politique que les classes ou groupes sociaux auxquels nous ont accoutumés les sciences sociales comme les statistiques d'État, ils proposent de ne considérer que des personnes en "situation ». S'ils privilégient les moments de crise (un conflit dans un atelier, par exemple) ou les actes de dénonciation (des plaintes déposées dans un commissariat, des lettres de protestation envoyées aux journaux), c'est que le compromis qui se construit localement révèle les tensions qui existent entre plusieurs modèles possibles de légitimation des positions individuelles et oblige à leur explicitation. Dans la dispute ou la dénonciation, chaque protagoniste mobilise un sens de ce qui est juste (par exemple, dans un conflit d'atelier: apprécier les gens selon leur compétence professionnelle, ou bien améliorer les conditions de travail, ou encore développer la démocratie syndicale, etc.). Boltanski et Thévenot empruntent à la philosophie politique les six «modèles » (au sens fort du terme) de justice qu'ils dénomment «cités » qui constituent les catégories d'une grammaire de la légitimation et du compromis et qui sont les ressources à la disposition des acteurs.

Ils offrent ainsi une alternative aux schémas d'analyse reçus et proposent une nouvelle représentation des rapports entre le particulier et le général, entre l'individuel et le collectif. Ils refusent aussi bien de considérer la totalité sociale comme une forme 
d'imposition qui pèse sur les acteurs que de doter ceux-ci d'une rationalité pure et parfaite indépendante de tout contexte. Le collectif apparaît comme le produit d'une construction datée et provisoire, comme le résultat d'un accord actif, mais temporaire et instable, qui engage pour un temps dans une configuration particulière les ressources critiques mobilisées par les acteurs selon les caractéristiques de la situation du moment. La stabilité et la durée de ces constructions collectives renvoient à la diversité des ressources mobilisables et à l'hétérogénéité des ressources effectivement mobilisées.

On voit bien, dans ce projet, comment le recours aux principes de légitimation consolide les manières de faire et les institutions sociales et organise les configurations interindividuelles particulières. On voit moins bien comment ces dernières affectent des modèles de légitimation qui semblent échapper à l'histoire et atteindre à l'universalité. L'échelle géographique et chronologique de l'analyse, qui vaut sans doute pour la situation contemporaine des sociétés occidentales, ne permet pas d'étudier les situations où bascule non seulement un régime local de justification (le moment où le principe démocratique l'emporte dans une usine sur le principe d'efficacité technique pour organiser le travail par exemple), mais où se modifie aussi la palette même des ressources mobilisables, des "cités » de référence à la disposition des acteurs : on rêve d'analyses semblables appliquées à des sociétés nées d'une conquête ou d'un métissage culturel. Même s'ils oublient cette dimension de l'histoire, les livres de Boltanski et de Thévenot proposent aux historiens des modifications de leur point de vue sur des aspects importants. Ils rappellent que chaque théorie, en sciences sociales, s'accompagne d'un type de temporalité pertinent et que celui-ci est en étroite relation avec les compétences dont la théorie considérée dote les acteurs. Ils suggèrent, en alternative à la chronique narrative ou à l'histoire de longue durée, l'intérêt analytique de la séquence brève, de la scène circonstanciée. Ils proposent enfin des procédures de totalisation qui ne procèdent pas par agrégation, mais sont issues de la compétence même des acteurs, des modalités de l'évaluation générale des situations dans lesquelles ils sont engagés, des formes de «montée en généralité » dont ils sont capables et qui constituent, ensemble, le lien social. La question du lien social court également depuis plusieurs années tout au long de l'œuvre de Jean-Pierre Dupuy ${ }^{22}$, construite sur deux idées fondamentales. D'une part, si les sciences sociales répondent si diversement à cette question, c'est que le lien qui unit les hommes est fondamentalement invisible :

La société tient ensemble « toute seule » c'est-à-dire au-delà ou plutôt en deça de la volonté et de la conscience des individus qui pourtant « l'agissent ».

D'autre part, il n'existe pas dans la société de point fixe exogène transcendant par rapport aux acteurs :

[...] le collectif humain prend pour point de repère extérieur quelque chose qui provient en fait de lui-même, par la composition des actions interdépendantes de ses membres.

Comment éclairer le mécanisme ? La panique, processus d'individualisation extrême où la société se pulvérise et où, dans le même mouvement, se recompose une nouvelle forme de totalisation, en donne par exemple le moyen. Toute une littérature de terrain apporte une confirmation (la panique appartient à la classe des représentations sociales autoréalisatrices) et une hypothèse : on passe d'une situation d'équilibre à la panique sans solution de continuité, et la décomposition de l'ordre naît de l'ordre lui-même. La psychologie des masses et la science économique, Freud et Walras, fournissent les éléments pour progresser. En situation de panique, la foule développe un processus d'imitation généralisée, où chacun copie chacun, contribuant à faire émerger un 
comportement général dont les caractères ne préexistent pas au système, mais qui paraissent extérieurs à lui. Sur le marché, les agents économiques rationalisent leurs comportements par rapport à un système de prix considéré comme déterminé par des facteurs objectifs extérieurs à eux, alors que c'est la combinaison de leurs décisions qui le font émerger.

Un pas encore, qui permettra de revenir vers l'histoire. Le spéculateur habile, si l'on en croit Keynes, est celui qui devine mieux que la foule ce que celle-ci va faire. La remarque conduit à souligner l'intérêt de l'analyse des jugements de convention et des processus de spéculation. En période normale, les références de chacun sont évidentes aux yeux de tous et les conduites se distribuent par rapport à ces conventions partagées. En période de crise et de perte du sens commun, la seule conduite rationnelle consiste à imiter les autres. De nouvelles références, apparemment objectives et extérieures au système d'acteurs, s'élaborent dans ce processus même. L'historien se trouve ici évidemment conforté dans l'utilité de ses études. Le mécanisme imitatif ouvre vers le nouveau, l'indéterminé ; il est potentiellement capable de faire émerger n'importe quel objet. Mais

[...] dans le temps effectif du processus, il se referme sur l'objet qu'il élit selon une dynamique auto-renforçante.

Il est le produit d'une histoire, et dépend d'un cheminement. Il n'est pas sûr pour autant qu'un historien doive être rassuré par de telles propositions. Si l'objet émergent n'est pas déterminé par déduction à partir de la structure formelle du jeu mais par son déroulement, que valent certaines manières de faire de l'histoire inscrites à l'enseigne du couple structure/conjoncture? Les façons traditionnelles de raconter ne valent pas plus. L'illusion de la pertinence tous azimuts d'une narration particulière s'applique tout autant au récit historique qu'à la biographie ${ }^{23}$.

Conventions pour un temps partagées: une nouvelle tendance de l'économie, qui a recours à l'histoire comme à une arme pour faire éclater le noyau dur de la théorie économique qui gravite autour du concept d'équilibre du marché concurrentiel pur et parfait, se fonde sur ce nouveau paradigme ${ }^{24}$. Sur la question de la totalité, l'économie des conventions pose, de façon systématique et d'une manière souvent neuve, beaucoup des questions auxquelles l'historien est affronté sur la question de la totalité sociale. J'en soulignerai quelques-unes. D'abord, la convention constitutive n'est pas le résultat d'un contrat positif $\mathrm{du}$ type rousseauiste, mais le produit d'un système d'interactions individuelles. Elle est à la fois le résultat d'actions particulières et elle constitue un cadre contraignant (et le plus souvent opaque) imposé par la "société et la tradition", pour reprendre les mots de Durkheim. Dans le processus de construction du social, elle remet en cause l'opposition simplifiée entre l'individu et les structures, entre la liberté et la contrainte, entre le passé et le présent. Ensuite, si la convention économique est une représentation collective (susceptible de prendre corps dans des organisations comme dans des règles de droit) qui permet la coordination des conduites individuelles, l'opposition réductrice entre les «faits» et les «représentations» (et la fuite méthodologique vers l'analyse des représentations pour elles-mêmes) se trouve disqualifiée. Les systèmes de connaissance, la construction de la mémoire, les processus d'apprentissage, l'information acquise ne constituent pas un simple cadre pour l'appréhension des phénomènes : ils les enregistrent et les instituent.

33 La variété des principes de coordination possibles crée un univers complexe. Elle détourne ainsi de la tentation de la pensée de la totalité par réduction à un principe unique d'explication. Le jeu ouvert entre plusieurs modes de coordination permet 
d'éviter tout déterminisme fonctionnaliste ou structuraliste. Il invite à réexaminer le type de rationalité supposée des acteurs. Il permet de ne pas réduire ces derniers à l'expression statistique de la cohérence des groupes auxquels ils appartiennent, sans renoncer à l'explication dynamique des conduites collectives comme ensemble de relations. Entre l'économique et le social, entre le culturel et l'économique, entre le social et le culturel, il permet de penser la société comme un système généralisé d'équivalences partielles et de tensions locales, dont les modalités sont décisives pour comprendre le changement. Car l'économie des conventions, enfin, s'inscrit résolument dans une perspective temporelle. Tout nouveau système de conventions y apparait déterminé par la contingence de son histoire. L'irréversibilité et la crise des conventions caractérisent le système économique. L'apprentissage et la rationalité procédurale sont le lot des acteurs 25 .

La solution sort-elle pour autant toute armée des propositions de quelques économistes? Il n'est pas question de le croire, mais les difficultés qu'ils résolvent mal sont, pour les historiens, autant d'incitations à renouveler leurs questionnaires et à préciser leurs analyses. La plupart des économistes inscrivent résolument les conventions du côté des pesanteurs temporelles. Routine, production répétée d'objets conformes à ses stipulations implicites ou explicites, règles qui permettent de réduire les effets du hasard: la convention tire en premier lieu sa stabilité du temps lui-même. Un tel soulignement a son prix. Les accords entre acteurs sont toujours susceptibles de présenter une figure particulière, mais ils s'inscrivent le plus souvent comme une variation localisée dans l'enveloppe plus large des conventions dominantes qu'ils peinent à atteindre. Il y a, à cela, trois motifs peut-être. D'une part, l'analyse accorde beaucoup plus d'attention à la manière dont la convention informe le compromis entre acteurs localisés qu'à la manière dont la suite des compromis renouvelle, jour après jour, dans sa succession même, les conventions. D'autre part, la graduation de l'échelle temporelle des économistes reste ici sommaire : entre la très longue durée des conventions et la succession des instants de leur mise à l'épreuve, il n'y a rien. Enfin, la conscience temporelle des acteurs est marquée par une dissymétrie: l'anticipation a pour eux, dans les modèles, plus d'importance que l'expérience. Tout se passe comme si les conventions avaient en charge le passé et les acteurs l'avenir.

Normes, valeurs, conventions sociales constituent des représentations collectives partagées et prennent corps dans des organisations, des institutions, des règles de droit. Elles apparaissent comme un cadre hérité du passé qui enserre et modèle les pratiques individuelles et collectives et semble ainsi tirer toute leur force de leur durée. Mais il est impossible d'imaginer des normes sociales inappliquées, des conventions économiques qu'aucun échange ne viendrait mettre à l'épreuve. Et au moment même de leur mise en acte, elles s'exposent au risque d'une réévaluation. Normes, valeurs, conventions sociales façonnent les accords locaux, mais se trouvent en retour façonnées par eux. Génératrices d'histoires elles se construisent et se défont, s'organisent sur des tensions temporelles, disposent de régimes d'historicité particuliers. À ce titre, il importe de questionner ces modèles à partir du point de vue de l'historien et de la pratique de l'histoire. C'est à un travail de ce type que ce texte voudrait inciter. 


\section{NOTES}

1. Bernard Lepetit, «C'est arrivé à Lournand », Médiévales, 21, automne 1991, p. 81-90.

2. Lucien Febvre, Combats pour l'histoire, Paris, 1953.

3. Id., La Terre et l'évolution humaine. Introduction géographique à l'histoire, Paris, 1922. Pour Ernest Labrousse, voir sa préface à Pierre Léon, La Naissance de la grande industrie en Dauphiné (fin du XVII -1869), Paris, 1954.

4. Jean-Yves Grenier, Séries économiques françaises (XVI ${ }^{e}-\mathrm{XVIII} I^{e}$ siècles), Paris, 1985.

5. Fernand Braudel, La Méditerranée et le monde méditerranéen à l'époque de Philippe II, Paris, 1949 ; Ernest Labrousse, La Crise de l'économie française à la fin de l'Ancien Régime et au début de la Révolution, Paris, 1944 (rééd. 1990).

6. Fernand Braudel," "Histoire et sciences sociales. La longue durée ", Annales ESC, 1958, p. 725-753.

7. Pierre Goubert, Beauvais et le Beauvaisis de 1600 à 1730. Contributions à l'histoire sociale de la France au XVII siècle, Paris, 1960.

8. Jean Heffer, et al., Outils statistiques pour les historiens, Paris, 1981.

9. Jean Bouvier, Initiation au vocabulaire et aux mécanismes économiques contemporains, Paris, 1969.

10. Pierre Chaunu, «L'économie. Dépassement et prospective ", in Jacques Le Goff et Pierre Nora, Faire de l'histoire, t. II, Paris, 1974, p. 51-73.

11. Emmanuel Le Roy Ladurie, «L'Histoire immobile », Annales ESC, 1974, p. 673-692.

12. Ordres et classes, Colloque de l'École normale de Saint-Cloud, 1967, Paris, 1974.

13. Jean-Claude Perrot, « Rapports sociaux et villes », Annales ESC, 1968, p. 241-268.

14. Id., Genèse d'une ville moderne. Caen au XVIII ${ }^{e}$ siècle, Paris, 1975.

15. Jacques Revel, «L'histoire au ras du sol », préf. in G. Lévi, Le Pouvoir au village. Histoire d'un exorciste dans le Piémont du XVII siècle, Paris, 1992.

16. Frédéric Le Play, La Méthode sociale. Abrégé des Ouvriers européens, présentation d'A. Savoye, Paris, 1989.

17. Clifford Geertz, Local Knowledge. Further Essays in Interpretative Anthropology, New York, 1983 ; Robert Darnton, The Great Cats Massacre, New York, 1984 ; G. Lévi, «I pericoli del geertzismo », Quaderni Storici, 1985, p. 269-277.

18. G. Lévi, L'Eredita immateriale. Carriera di un esorcista nel Piemonte del seicento, Turin, 1985 ; M. Gribaudi, Mondo operaio e mito operaio. Spasi e percorsi sociali a Torino nel primo novecento, Turin, 1987 ; S. Cerruti, La Ville et les métiers. Naissance d'un langage corporatif (Turin, XVII ${ }^{e}$-XVIII ${ }^{e}$ siècles), Paris, 1988.

19. Bernard Lepetit, Les Villes dans la France moderne (1740-1840), Paris, 1988.

20. Paul-André Rosental, "Construire le macro par le micro : Frederik Barth et la microstoria ", Anthropologie contemporaine et anthropologie historique, Colloque de l'École des hautes études en sciences sociales, Marseille, 24-26 septembre 1992 (multigraphié).

21. Luc Boltanski, L'Amour et la justice comme compétences. Trois essais de sociologie de l'action, Paris, 1990 ; Luc Boltanski et Louis Thévenot, De la justification. Les économies de la grandeur, Paris, 1991.

22. Jean-Pierre Dupuy, Ordres et désordres. Enquête sur un nouveau paradigme, Paris, 1982. Id., La Panique, Paris, 1991.

23. Jean-Claude Passeron, Le Raisonnement sociologique. L'espace non-poppérien du raisonnement naturel, Paris, 1991 ; Bernard Lepetit, « Une logique du raisonnement historique », Annales ESC, 
24. "L'économie des conventions", Revue économique, mars 1989 ; Jacques Lesoume, Économie de l'ordre et du désordre, Paris, 1991.

25. R. Boyer, B. Chavance, O. Godard, Les Figures de l'irréversibilité en économie, Paris, 1991. 